\title{
Implications for minimal supersymmetry from grand unification and the neutralino relic abundance
}

\author{
R.G. Roberts \\ Rutherford Appleton Laboratory, Chilton, Didcot, Oxon OX11 OQX, UK \\ and \\ Leszek Roszkowski ${ }^{1}$ \\ Randall Physics Laboratory, University of Michigan, Ann Arbor, MI 48190, USA
}

Received 25 January 1993; revised manuscript received 17 April 1993

Editor: H. Georgi

\begin{abstract}
We examine various predictions of the minimal supersymmetric standard model coupled to minimal supergravity. The previously considered predictions of the model are now extended to include the bottom quark mass and the neutralino relic density which in turn provide stringent bounds on the scale parameters. We find a remarkable consistency among several different constraints only in the region of low-energy supersymmetry, thus avoiding the use of the fine tuning criterion. We find all supersymmetric particle masses preferably within the reach of future supercolliders (LHC and SSC). The requirement that the neutralino be the dominant component of (dark) matter in the flat Universe provides a lower bound on the spectrum of supersymmetric particles beyond the reach of LEP, and most likely also the Tevatron and LEP 200.
\end{abstract}

\section{Introduction}

The minimal extension of the standard model [1] which corresponds to a softly-broken supersymmetric $\mathrm{SU}(3) \times \mathrm{SU}(2)_{L} \times \mathrm{U}(1)_{Y}$ at the GUT scale $M_{X}$ where the gauge couplings unify (as recently confirmed by LEP [2]) provides a very attractive and economic description of physics beyond the standard model. It is possible to specify a small number of parameters at the unification scale and the low energy effective theory is then determined simply by the radiative corrections. In particular the spontaneous breaking of electroweak symmetry is radiately generated due to the presence of supersymmetry soft-breaking terms through the mass squared of one of the two Higgs doublets being driven negative at the scale $Q \simeq \mathrm{O}\left(m_{Z}\right)$ by the Yukawa top quark coupling [3]. In terms of the starting parameters at the GUT scale a detailed spectrum of the super-

1 E-mail address: leszek@leszek.physics.lsa.umich.edu. symmetric (SUSY) states is completely determined. Even in the simplest SUSY GUT models, like SU(5), one meets some uncertainty related to the presence of the superheavy states around the GUT scale [46]. The corresponding threshold corrections depend on which unified group or superstring scenario the minimal SUSY model is embedded into. These model dependent corrections would weaken the predictive power of the theory and, as in a previous paper [6], we ignore them. Similarly, the constraint coming from the limits on the proton decay [7] depends on the choice of a specific GUT model and will not be discussed here.

In this letter, we consider the minimal supergravity model without adhering to any specific GUT. We treat corrections from supersymmetric states above $m_{Z}$ with particular care [6]. Our goal is to explore to what extent the various physical quantities, like $\alpha_{\mathrm{s}}$, $m_{t}, m_{b}$, and the relic density, resulting from GUTscale assumptions are consistent with each other and 
with the notion of low-energy supersymmetry. We do not use the fine tuning criterion as a constraint. We extend the previous analysis [6] to properly include predictions for the bottom quark mass. We also calculate the relic abundance of the lightest neutralino $\chi$ which is usually the lightest supersymmetric particle (LSP) of the model. The neutralino LSP has long been identified [8] as one of the leading candidates for dark matter in the Universe $[9,10]$. It is neutral, weakly interacting, stable (if $R$-parity is valid) particle and its relic density is typically consistent with present cosmological expectations. We examine the predictions for $\chi$ from the minimal supersymmetric standard model (MSSM) and compute the annihilation cross sections which requires the detailed knowledge of the whole SUSY spectrum. Consequently we can relate values of the neutralino relic abundance to values of the parameters $m_{1 / 2}, m_{0}, \mu_{0}$ - the common gaugino mass, the common scalar mass and the higgsino mass at $M_{X}$. The lower limit on the age of the Universe provides an upper bound on the relic abundance of matter, and in particular of dark matter which is believed to be a dominant mass component of the Universe. We can therefore use the dark matter abundance constraint to derive bounds on the ranges of $m_{1 / 2}, m_{0}, \mu_{0}$ and in turn get constraints on the masses of all the SUSY particles.

We combine the dark matter constraint with experimental constraints on $\alpha_{\mathbf{s}}\left(m_{Z}\right), m_{t}$ and $m_{b}$ and examine the consistency of trying to satisfy several of these constraints simultaneously. We conclude that one can indeed achieve such consistency quite naturally. More interestingly, we find that this happens for the ranges of the fundamental parameters $m_{1 / 2}, m_{0}, \mu_{0}$, and thus also masses of the supersymmetric particles, all preferably within the few hundred $\mathrm{GeV}$ mass range. We thus find that requiring consistency between the criteria listed above leads us towards truly low-energy supersymmetry well within the reach of the SSC and the LHC. We also find that the favoured region does not require any significant amount of fine tuning, expected to avoid the mass hierarchy problem. We emphasize that our results are consistent with, but not constrained by, the fine tuning criterion.

We also find that the resulting supersymmetric spectra lie typically above the reach of LEP, the Tevatron, and LEP 200. The lower limit on supersymmetric particle masses comes from the dark matter constraint as will be discussed in section 3 .

The LSP for which we find sufficiently large values of the relic abundance to explain at least DM in the galactic halos $\left(\Omega h_{0}^{2} \gtrsim 0.025\right)$ invariably comes out to be the lightest neutralino, in fact of gauginotype (bino-like), consistent with the conclusions of some previous analyses [11-13]. It was first argued in ref. [11] that a gaugino-like LSP makes a unique candidate for DM. Higgsino-like LSP is somewhat disfavoured as it corresponds to a high scale of supersymmetry breaking, typically exceeding $1 \mathrm{TeV}$ [11]. More recently, it has been shown [13,14] that for the higgsino-like neutralinos additional effects (coannihilation with the next-to-lightest neutralino and the lightest chargino, see section 3 ) have a dramatic effect of reducing the LSP relic abundance below any interesting level. Here we find that in addition higgsinolike LSPs are largely excluded by the current lower bound on the mass of the top quark.

Overall, the LSP relic abundance constraint, combined with the other physical constraints, narrows down the allowed ranges of $m_{1 / 2}, m_{0}, \mu_{0}$ considerably. We find that the region $m_{1 / 2} \gg m_{0}$ is excluded by the lower bound on the top mass, while in the region $m_{1 / 2} \ll m_{0}$ the LSP relic abundance is too large $\left(\Omega h_{0}^{2}>1\right)$. Furthermore, the requirement that the LSP provide enough missing mass in the flat $(\Omega=1)$ Universe can be fulfilled only in a relatively narrow band of comparable values of $m_{1 / 2}$ and $m_{0}$ and for $1<\mu_{0} / m_{0} \lesssim$ a few.

In the next section we briefly review the procedure used in deriving the low-energy spectrum from a limited number of basic parameters at the GUT scale. In section 3 we demonstrate the importance of the neutralino relic density constraint and compare it with other constraints on the parameter space. We conclude with final remarks in section 4 .

\section{Solutions of the MSSM}

We follow the procedure of extracting solutions of the MSSM consistent with the unification of the gauge couplings at the scale $M_{X}$ developed in ref. [6]. This method takes properly into account the threshold effects on the running of the gauge couplings due to supersymmetric states above $m_{Z}$. Each solution is characterised by the values of a small number of param- 
eters at $Q=M_{X}: m_{1 / 2}, m_{0}, \mu_{0}, A_{0}$, and $B_{0}$ which determine the masses of the SUSY spectrum at low energies. Of the Yukawa couplings, in the evolution of the SUSY masses we keep only $h_{t}$. Its value $h_{t 0}$ at $M_{X}$ must be precisely adjusted to reproduce the correct electroweak symmetry breaking, i.e. the value of $M_{Z}$, thus leading to a certain degree of "fine tuning", as pointed out in ref. [6].

This insistence on "naturalness" is essentially a theoretical constraint which rules out large values of $m_{1 / 2}$, $m_{0}, \mu_{0}$ and leads to a narrow set of solutions. While it is suggestive, not only is it not a uniquely defined quantity [15] but also its validity depends on ones personal taste. Of similarly restricted value are often made assumptions that the resulting mass spectra should be below $1 \mathrm{TeV}$. In the present analysis we shall not use the naturalness criterion to restrict the allowed parameter space, in contrast to all recent analyses. Instead, we shall see that a combination of "observed" limits on $\alpha_{\mathrm{s}}, m_{b}$, and the relic abundance of dark matter effectively replace this theoretical criterion so that one can arrive at a restricted set of solutions in the low-energy range $\leqslant \mathrm{O}(1 \mathrm{TeV})$ purely on phenomenological grounds.

In calculating $m_{b}$ we make the usual assumption that the Yukawa couplings $h_{b 0}=h_{\tau 0}$ at $M_{X}$. Below we discuss the validity of this assumption.

We illustrate the various constraints by showing the values of $m_{t}$ and $m_{b}$ in fig. 1a and $\alpha_{s}\left(m_{Z}\right)$ and fine tuning $c$ in fig. $1 \mathrm{~b}$ as a function of $m_{1 / 2}$ and $m_{0}$ for a fixed ratio $\mu_{0} / m_{0}=2$. The variation with $\mu_{0} / m_{0}$ will be discussed later. The regions marked CDF and LEP are excluded by the CDF searches for the top $\left(m_{t} \gtrsim 91 \mathrm{GeV}\right)$ and the LEP searches for charginos $\left(m_{\chi_{1}^{ \pm}} \gtrsim 46 \mathrm{GeV}\right)$, respectively. We see from fig. 1a that the current "experimental" value for $m_{b}$ (in the $\overline{\mathrm{MS}}$ scheme), $m_{b}\left(2 m_{b}\right)=4.25 \pm 0.1 \mathrm{GeV}$ [16], implies a rather heavy top quark $\left(m_{t} \gtrsim 150 \mathrm{GeV}\right)$ for the values of the input parameters $m_{1 / 2}, m_{0}$ and $\mu_{0}$ roughly within the $1 \mathrm{TeV}$ limit. On the other hand, beyond that range the resulting value of $m_{b}$ is consistent with $m_{t} \lesssim 150 \mathrm{GeV}$.

Very large values of the input mass parameters are, however, ruled out by a lower bound on $\alpha_{\mathbf{s}}$, as shown in fig. 1 b. Several recent analyses have arrived at consistent ranges: $\alpha_{\mathrm{s}}=0.122 \pm 0.010$ [17] (based on analysis of jets at LEP), $\alpha_{\mathrm{s}}=0.117 \pm 0.007$ [18], and $\alpha_{\mathrm{s}}=0.125 \pm 0.002$ (expt.) \pm 0.009 (theor.) [19]. Requiring conservatively $\alpha_{\mathrm{s}}>0.110$ restricts the input mass parameters roughly below $5 \mathrm{TeV}$.

We also illustrate in fig. $1 \mathrm{~b}$ the amount of fine tuning [6] related to the presented case. Demanding $c \lesssim$ $\mathrm{O}(10)$ would force one to consider only values of $m_{1 / 2}, m_{0}$ up to a few hundred $\mathrm{GeV}$ [6]. Clearly larger values of $m_{1 / 2}$ and $m_{0}$, and correspondingly lower values of $\alpha_{s}(\lesssim 0.114)$ are strongly disfavoured by the fine-tuning criterion but cannot be strictly ruled out.

To summarise so far, the solutions obtained for the MSSM with the inclusion of electroweak symmetry allow a fairly restricted region of the parameters $m_{1 / 2}, m_{0}$ within the range of a few $\mathrm{TeV}$, the upper bound coming from the lower bound on $\alpha_{s}$. We will comment on the restrictions on the ratio $\mu_{0} / m_{0}$ and the role of of other assumptions later.

\section{The neutralino relic abundance}

The knowledge of the whole mass spectrum of both the ordinary and supersymmetric particles allows one to reliably compute the relic abundance of the lightest supersymmetric particle (LSP) as a candidate for the dark matter in the Universe.

At the outset we note that, in the parameter space not already excluded by LEP and CDF, we find that it is the lightest of the four neutralinos that is always the LSP. Another potential candidate for the LSP, the sneutrino, has now been constrained by LEP to be heavier than about $42 \mathrm{GeV}$ and, if it were the LSP, its contribution to the relic abundance would be on the order of $10^{-4}$, and thus uninterestingly small. In the analysis presented here, the sneutrino is typically significantly heavier than the lightest neutralino. Typically, it is not even the lightest sfermion.

The actual procedure of calculating the relic abundance has been adequately described in the literature and will not be repeated here. We use the technique developed in ref. [20] which allows for a reliable (except near poles and thresholds) computation of the thermally averaged annihilation cross section in the non-relativistic limit and integrating the Boltzmann equation.

In the early Universe the LSP pair annihilated into ordinary matter with total mass not exceeding $2 m_{x}$. 

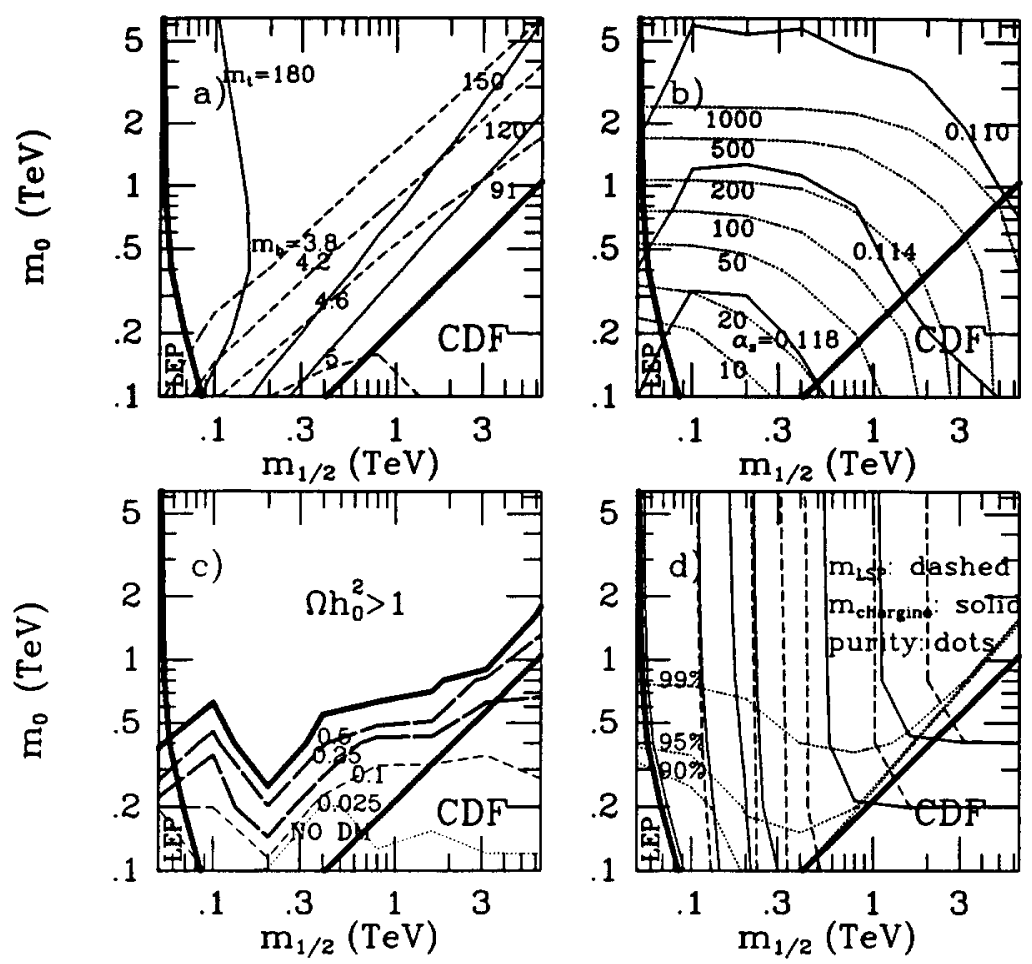

Fig. 1. In the plane $\left(m_{1 / 2}, m_{0}\right)$ for the fixed ratio $\mu_{0} / m_{0}=2$ we show: in window (a) the mass contours of the top and the bottom quarks (solid and short-dashed lines, respectively); in window (b) the contours of $\alpha_{S}\left(m_{Z}\right)$ (solid) and the measure $c$ of fine-tuning (dots), as discussed in the text; in window (c) the relic abundance $\Omega h_{0}^{2}$ of the LSP; and in window (d) the mass contours of the LSP (solid) and the lightest chargino (dashed) at 50, 100, 150, 200, 500, and 1000 GeV, starting from left, and the contribution (dots) of the bino to the LSP composition (bino purity, as discussed in the text). In all windows thick solid lines delineate regions experimentally excluded by the CDF (marked CDF) where $m_{t}<91 \mathrm{GeV}$ and by the LEP experiments (LEP) where the lightest chargino is lighter than $46 \mathrm{GeV}$. In window (c) we also mark by $\Omega h_{0}^{2}>1$ the region cosmologically excluded (too young Universe). The thin band between the thick dashed lines in window (c) corresponds to the flat Universe $(\Omega=1)$, as discussed in the text. In window (d) the region excluded by CDF almost coincides with the bino purity of $50 \%$ or less.

In calculating the LSP relic density we include all possible final states.

Generally one considers $\Omega h_{0}^{2}>1$ as incompatible with the assessed lower bound of about $10 \mathrm{Gyr}$ on the age of the Universe or, in more popular terms, as corresponding to too much mass in the Universe [10]. Many astrophysicists strongly favour the value $\Omega=1$ (or very close to one), corresponding to the flat Universe, either because of cosmic inflation or for aesthetical reasons. Moreover, there is growing evidence that, on a global scale, the mass density indeed approaches the critical density, as well as that most of the matter in the Universe is non-shining and nonbaryonic [10]. If one assumes that the LSP is the dom- inant component of dark matter in the flat $(\Omega=1)$ Universe then one typically expects

$0.25 \lesssim \Omega h_{0}^{2} \lesssim 0.5$

where the biggest uncertainty lies in our lack of knowledge of the Hubble parameter $h_{0}$ to better than a factor of two. As we will see shortly, varying somewhat the bounds in eq. (1) will not significantly alter our conclusions.

We present in fig. $1 \mathrm{c}$ the relic abundance of the LSP and compare it with the other results shown before in figs. $1 \mathrm{a}$ and $1 \mathrm{~b}$. Several features can be immediately noticed. 
Firstly, most of the region corresponding to larger values of $m_{0}$ (roughly $m_{0} \gtrsim 500 \mathrm{GeV}$ ) is cosmologically excluded as it corresponds to $\Omega h_{0}^{2}>1$. The relic abundance generally decreases with decreasing $m_{0}$ reaching very low values of $\Omega h_{0}^{2}(0.025$, or less) for $m_{0}$ roughly below $200 \mathrm{GeV}$, especially for $m_{1 / 2}>$ $m_{0}$. The cosmological bound becomes much weaker around the direction $m_{0} \simeq \frac{1}{3} m_{1 / 2}$ thus allowing for $m_{1 / 2}$ to lie in the range of several $\mathrm{TeV}$. In that region, however, the mass of the bottom is significantly larger than the favoured range of $4.25 \pm 0.1 \mathrm{GeV}$. The combination of these two constraints thus restricts both $m_{1 / 2}$ and $m_{0}$ to the truly low-energy range of several hundred $\mathrm{GeV}$.

Next we stress that the region favoured by cosmology, eq. (1), takes a shape of a relatively narrow band running roughly parallel to the area excluded by $\Omega h_{0}^{2}>1$. The contour $\Omega h_{0}^{2}=0.1$ shows how quickly $\Omega h_{0}^{2}$ decreases with decreasing $m_{0}$. It also limits from below the region where the LSP relic abundance is reasonably large.

It is interesting to see what mass and compositions of the LSP correspond to its relic abundance favoured by cosmology. We remind the reader that, in minimal supersymmetry, the lightest neutralino and its three heavier partners $\chi_{i}^{0}(i=1, \ldots, 4)$ are the physical (mass) superpositions of higgsinos $\widetilde{H}_{1}^{0}$ and $\widetilde{H}_{2}^{0}$, the fermionic partners of the neutral Higgs bosons, and of two gauginos $\widetilde{B}^{0}$ and $\widetilde{W}_{3}^{0}$, the fermionic partners of the neutral gauge bosons,

$\chi \equiv \chi_{1}^{0}=N_{11} \widetilde{W}^{3}+N_{12} \widetilde{B}+N_{13} \widetilde{H}_{1}^{0}+N_{14} \widetilde{H}_{2}^{0}$.

In distinguishing the gaugino-like and higgsino-like regions it is convenient to use the gaugino purity $p=$ $N_{11}^{2}+N_{12}^{2}$. In particular, the LSP is almost a pure bino where $p_{\text {bino }} \equiv N_{12}^{2}$ is close to one. In fig. 1d we show the bino purity of the LSP. (The gaugino purity is almost identical.) Remarkably, we find that the band favoured by cosmology corresponds to the LSP being almost a pure bino $(\sim 95 \%)$ up to very large values of $m_{1 / 2}$. We also find that higgsino-like LSPs are incidentally almost entirely excluded by the lower bound on the top quark of $91 \mathrm{GeV}$. (The contour of equal gaugino and higgsino contributions almost coincides with the contour $m_{t}=91 \mathrm{GeV}$.) It was also noticed in ref. [13] that for a heavy top constraints from radiative gauge symmetry breaking exclude higgsinolike LSPs. (With the expectation for $m_{t}$ to be actually much heavier than $91 \mathrm{GeV}$ a larger cosmologically uninteresting region is likely to be ruled out.) The LSP mass contours are almost vertical in the gaugino region with $m_{\chi}$ growing with $m_{1 / 2}$, and almost horizontal in the higgsino region with $m_{\chi}$ increasing with $m_{0}$. Again, the lines meet in the narrow sub-diagonal region around $m_{0} \simeq \frac{1}{3} m_{1 / 2}$ where the LSP is both a gaugino and a higgsino.

By comparing figs. 1c and $1 \mathrm{~d}$ we find no "purely" cosmological upper bound on the mass of the LSP, in some contrast to ref. [13]. This is simply because we never find the LSP to be a pure gaugino. On the other hand, by requiring in addition that $m_{b}$ be in the favoured range leads us to an upper bound of about $500 \mathrm{GeV}$ on the mass of the effectively bino-like LSP, in general agreement with ref. [13].

Since higgsino-like LSPs in our analysis not only give very little DM but also are practically excluded by the CDF top searches, we need not worry about the additional effect of the higgsino-like LSP "coannihilation" [21] with the next-to-lightest neutralino and the lightest chargino which has been recently shown to significantly reduce the LSP relic density $[13,14]$. We have explicitly verified that all solutions for which co-annihilation of the LSP with $\chi_{2}$ and $\chi_{1}^{ \pm}$is important lie in the region excluded by $m_{t} \geqslant 91 \mathrm{GeV}$. Thus neglecting the effects of co-annihilation is justified.

The LSP relic abundance in the allowed region is mostly dominated by its annihilation into fermionic final states, although in a few cases the Higgs final states contributes comparably. We thus do not expect that the radiative corrections to the Higgs masses due to the heavy top [22] would noticeably modify our results [12]. We also found that the lightest sfermion is either $\widetilde{t}_{\mathbf{R}}$ or $\widetilde{l}_{R}$ (in agreement with ref. [13]) except in the (mostly excluded by LEP) region of small $m_{0}$ and $m_{1 / 2}$ where it is the sneutrino.

We have illustrated that by combining the cosmological constraint with the mass contours of the top and the bottom quarks one selects the low-energy region of a few hundred $\mathrm{GeV}$. It is appropriate to comment here how this conclusion depends on varying the ratio $\mu_{0} / m_{0}$ and including non-zero values of $A_{0}$ and $B_{0}$. When the ratio $\mu_{0} / m_{0}$ is decreased, the relic abundance contours generally move towards larger values of $m_{0}$ as do the contours for $m_{t}$ and $m_{b}$. For $\mu_{0} / m_{0}=$ 1 the favoured range of the bottom quark mass of 
about $4.25 \mathrm{GeV}$ lies entirely within the cosmologically excluded region $\Omega h_{0}^{2}>1$. On the other hand as $\mu_{0} / m_{0}$ increases, $m_{b}=4.25$ takes us to a region of larger $m_{1 / 2}$ and lower $m_{0}$ while the contours relic abundance remain relatively unchanged. The area consistent with the constraints of $m_{b}, m_{t}$ corresponds to lower values of the relic abundance, $\Omega h_{0}^{2} \lesssim 0.25$. If we increase $\mu_{0} / m_{0}$ still further the region of overlap for the constraints of $m_{b}, m_{t}$, and $\Omega=1$ shrinks and leads us the region of larger $m_{1 / 2}$ and much smaller $\alpha_{s}$. We thus conclude that the combination of all the above constraints selects the range $1 \leqslant \mu_{0} / m_{0} \lesssim$ a few. Next, allowing $A_{0}$ and $B_{0}$ to be non-zero has a primary impact on $\tan \beta$ mostly, and thus could be used to reduce by some $10 \%$ the mass of the top, which, as we pointed out above, comes out rather heavy, $m_{t} \gtrsim 150 \mathrm{GeV}$. Should the top quark be discovered at a much lower mass range, one would presumably be forced to relax some of the stringent assumptions of the minimal supergravity model considered here. For example, one may contemplate relaxing the assumption $h_{b 0}=h_{\tau 0}$ at $M_{X}$, as also suggested by some superstring scenarios.

We now concentrate on the low-energy range of the input mass parameters selected above by combining the constraints on $m_{b}, \alpha_{\mathrm{s}}$, and the cosmologically allowed region. This is shown in fig. 2. We see that the region where the LSP gives the dominant contribution to the matter density of the flat Universe (marked $\Omega=1$ ) crosses the estimated value of the bottom quark mass $\left(m_{b}=4.25 \pm 0.1 \mathrm{GeV}\right)$ for $m_{t}$ broadly between $160 \mathrm{GeV}$ and $180 \mathrm{GeV}$. Remarkably, this happens for $150 \mathrm{GeV} \lesssim m_{1 / 2}, m_{0} \lesssim 400 \mathrm{GeV}$, the range also strongly favoured by constraints from $\alpha_{\mathrm{s}}$ and fine tuning.

In the selected range all the Higgs bosons, squarks and sleptons, as well as the gluino, are significantly lighter than $1 \mathrm{TeV}$ and thus are bound to be found at the LHC and SSC.

On the other end, there is a strong expectation that the (LSP) dark matter dominates the mass of the flat Universe. (In minimal supersymmetry no other particle can even significantly contribute to the missing mass.) It then implies a significant lower bound on the spectrum of supersymmetric particle masses. We see from figs. 1d and 2 that the LSP masses favoured by all the constraints lie in the range

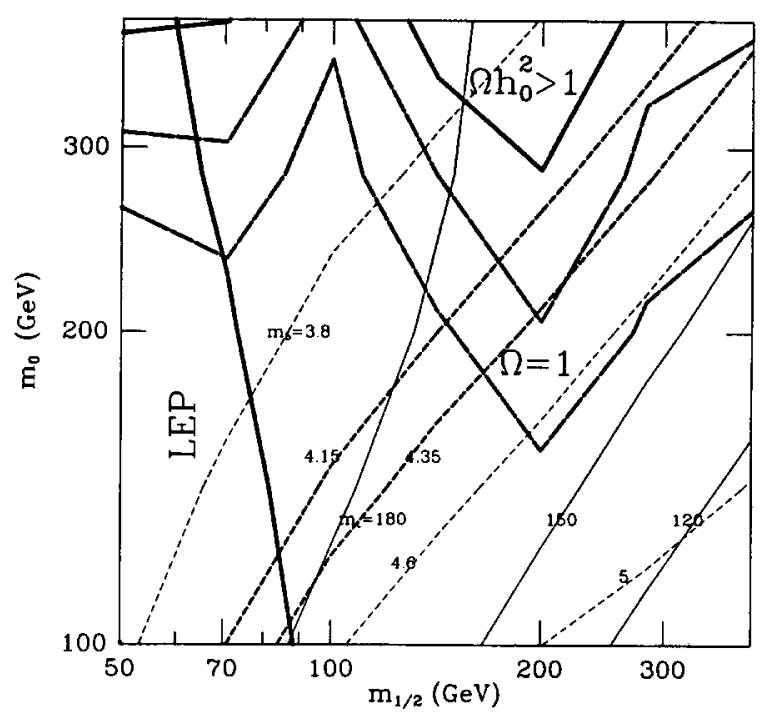

Fig. 2. We show a blow-up of the down-left portion of the plane $\left(m_{1 / 2}, m_{0}\right)$ from the previous figure for the same fixed ratio $\mu_{0} / m_{0}=2$. We combine the mass contours of the top and the bottom quarks with the ones of the LSP relic mass density. We use the same textures as in fig. 1 but we also show (two medium-thick short-dashed lines) the contours $m_{b}=4.15 \mathrm{GeV}$ and $4.35 \mathrm{GeV}$ which reflect the currently favoured range of the mass of the bottom quark (see text). We see that they cross the cosmologically favored region (thick long-dashed lines) marked $\Omega=1$ at roughly 150 $\mathrm{GeV} \lesssim m_{1 / 2}, m_{0} \lesssim 400 \mathrm{GeV}$ and for $m_{t}$ broadly between 150 and $180 \mathrm{GeV}$.

$60 \mathrm{GeV} \lesssim m_{\chi} \lesssim 200 \mathrm{GeV}$,

the upper limit being also expected in the minimal supersymmetric model $[11,23]$ on the basis of naturalness. Similarly, we find

$150 \mathrm{GeV} \lesssim m_{\chi_{1}^{ \pm}} \lesssim 300 \mathrm{GeV}$,

$200 \mathrm{GeV} \lesssim m_{\tau} \lesssim 500 \mathrm{GeV}$,

$250 \mathrm{GeV} \lesssim m_{\tilde{q}} \lesssim 850 \mathrm{GeV}$,

$350 \mathrm{GeV} \lesssim m_{\tilde{g}} \lesssim 900 \mathrm{GeV}$.

The heavy Higgs bosons are roughly in the mass range between $250 \mathrm{GeV}$ and $700 \mathrm{GeV}$. Of course, lower values of all these masses correspond to less fine tuning and larger values of $\alpha_{\mathrm{s}}$. The lightest Higgs boson treelevel mass invariably comes out close to $m_{Z}$; its oneloop-corrected value [22] is then roughly in the range 
120 to $150 \mathrm{GeV}$. By comparing figs. $1 \mathrm{~b}$ and 2 we also find $0.116 \lesssim \alpha_{\mathrm{s}}\left(m_{Z}\right) \lesssim 0.120$. We thus arrive at a cosmological upper bound on $\alpha_{\mathrm{s}}$. (Larger values of $\alpha_{\mathrm{s}}$ are also disfavoured by considering threshold corrections at the GUT scale [5].)

Thus, if the LSP is indeed the dominant component of DM in the flat Universe, supersymmetric particles are probably beyond the reach not only of LEP but also the Tevatron and LEP $200[24,12,13,25]$. We note, on the other hand, that smaller ranges of supersymmetric particles are not firmly excluded but would correspond to the LSP contributing only a fraction of the critical density. We believe that a fully exhaustive search of the whole parameter space might weaken the derived bounds by some $10 \%$. For example, Drees and Nojiri [13] have found in certain extreme cases (rather large values of $A_{0}$ ) squarks even somewhat lighter that $200 \mathrm{GeV}$ and a lower limit $m_{0}>40 \mathrm{GeV}$, although they considered only a fixed value of $M_{X}$ and neglected the effect of multiple mass thresholds. We find that the condition $\Omega=1$ requires in our case significantly larger values of $m_{0}\left(m_{0} \gtrsim 150 \mathrm{GeV}\right)$, in agreement with refs. [12,25]. However, we do not consider it to be in contradiction with the mentioned results of ref. [13] but a reflection of somewhat different assumptions at the GUT scale and methods of deriving the supersymmetric mass spectra. We do not expect that the procedure adopted here would produce substantially modified results by including the full one-loop effective potential or by performing a finer search of the parameter space.

\section{Conclusions}

Our primary conclusion is that one can restrict the parameter space of the MSSM in the context of a unified theory to a the low-energy region of less than about $1 \mathrm{TeV}$ without using the fine tuning criterion. In order to achieve this one needs to simultaneously consider the bounds on $\alpha_{s}, m_{b}$, and the allowed relic density in the Universe $\left(\Omega h_{0}^{2}<1\right)$. The resulting supersymmetric mass spectra consistent with all the above constraints lie within the discovery limits of the SSC, and for the most part also LHC.

We do find that cosmologically attractive LSP is almost purely bino-like $(\sim 95 \%)$ and lies in the range $60 \mathrm{GeV} \lesssim m_{\chi} \lesssim 200 \mathrm{GeV}$. Moreover, if the LSP dom- inates the dark matter in the (flat) Universe then the expected ranges of chargino, slepton and Higgs boson (with a possible exception of the lightest scalar) masses lie beyond the reach of LEP 200 . This was first noticed in ref. [24] and confirmed in refs. [12,13,25]. The associated ranges of gluino and squark masses then exceed the reach of the Tevatron.

Generally, we find it very reassuring that, in the simplest and most economic supersymmetric scenario, a careful analysis of the implications of several different (and independent) constraints, including the DM constraint, which result from the grand unification conditions, leads to a supersymmetric spectrum accessible to the next generation of accelerators.

\section{Acknowledgement}

We thank Graham Ross and Gordon Kane for inspiration and numerous discussions. This work was supported in part by the US Department of Energy.

\section{References}

[1] For reviews, see, e.g., H.-P. Nilles, Phys. Rep. 110 (1984) 1

L.E. Ybáñez and G.G. Ross, CERN preprint CERNTH.6412/92 (February 1992), in: Perspectives in Higgs physics, ed. G. Kane, to appear;

H.E. Haber and G.L. Kane, Phys. Rep. 117 (1985) 75.

[2] P. Langacker and M.-X. Luo, Phys. Rev. D 44 (1991) 817;

U. Amaldi, W. de Boer and H. Fürstenau, Phys. Lett. B 260 (1991) 447;

F. Anselmo, L. Cifarelli, A. Peterman and A. Zichichi, Nuovo Cim. 104A (1991) 1817; 105A (1992) 581;

J. Ellis, S. Kelley and D.V. Nanopoulos, Phys. Lett. B 260 (1991) 131.

[3] L.E. Ibáñez and G.G. Ross, Phys. Lett. B 110 (1982) 215 ;

K. Inoue, A. Kakuto, H. Komatsu and S. Takeshita, Progr. Theor. Phys. 68 (1982) 927;

L. Alvarez-Gaumé, M. Claudson and M. Wise, Nucl. Phys. B 207 (1982) 96;

J. Ellis, D.V. Nanopoulos and K. Tamvakis, Phys. Lett. B 121 (1983) 123.

[4] R. Barbieri and L.J. Hall, Phys. Rev. Lett. 68 (1992) 752 ;

P. Langacker and N. Polonsky, University of Pennsylvania preprint UPR-0513T (1992);

K. Hagiwara and Y. Yamada, KEK preprint KEK-TH331 (May 1992); 
V. Barger, M.S. Berger and P. Ohmann, Madison preprint MAD-PH-711 (September 1992).

[5] A. Faraggi, B. Grinstein and S. Meshkov, SSCL preprint SSCL-Preprint-126 (August 1992).

[6] R.G. Roberts and G.G Ross, Nucl. Phys. B 377 (1992) 571 .

[7] J. Ellis, D.V. Nanopoulos and S. Rudaz, Nucl. Phys. B 202 (1982) 43;

R. Arnowitt and P. Nath, Phys. Rev. Lett. 69 (1992) 725; Phys. Lett. B 287 (1992) 89; preprints NUB-TH3048-92, NUB-TH-3055-92, NUB-TH-3056-92;

J. Hisano, H. Murayama and T. Yanagida, Tohoku University preprint TU-400 (July 1992).

[8] J. Ellis, J.S. Hagelin, D.V. Nanopoulos, K.A. Olive and M. Srednicki, Nucl. Phys. B 238 (1984) 453.

[9] S.M. Faber and J.S. Gallagher, Annu. Rev. Astron. Astrophys. 17 (1979) 135;

J.R. Primack, B. Sadoulet and D. Seckel, Annu. Rev. Nucl. Part. Sci. B 38 (1988) 751;

V. Trimble, Annu. Rev. Astron. Astrophys. 25 (1987) 425 .

[10] E. Kolb and M. Turner, The early Universe (AddisonWesley, Reading, MA, 1989).

[11] L. Roszkowski, Phys. Lett. B 262 (1991) 59.

[12] J. Ellis and L. Roszkowski, Phys. Lett. B 283 (1992) 252.

[13] M. Drees and M. Nojiri, DESY preprint DESY 92-101 (July 1992).

[14] S. Mizuta and M. Yamaguchi, Tohoku University preprint TU-409 (July 1992).

[15] B. de Carlos and J.A. Casas, CERN preprint CERNTH.6835/93 (March 1993).
[16] J. Gasser and H. Leutwyler, Phys. Rep. 87 (1982) 77; S. Narison, Phys. Lett. B 216 (1989) 191.

[17] G. Altarelli, CERN preprint CERN-TH-6623/92 (August 1992).

[18] I. Hinchliffe, talk, in: Proc. Rencontres de Moriond (1993), to appear.

[19] P. Langacker, University of Pennsylvania preprint UPR-0555T (March 1993), presented at TASI-92 (Boulder, CO, June 1992).

[20] M. Srednicki, R. Watkins and K.A. Olive, Nucl. Phys. B 310 (1988) 693 .

[21] K. Griest and D. Seckel, Phys. Rev. D 43 (1991) 3191.

[22] Y. Okada, M. Yamaguchi, and T. Yanagida, Prog. Theor. Phys. 85 (1991) 1; Phys. Lett. B 262 (1991) 54 ;

H.E. Haber and R. Hempfling, Phys. Rev. Lett. 66 (1991) 1815

J. Ellis, G. Ridolfi and F. Zwirner, Phys. Lett. B 257 (19991) 83; B 262 (1991) 477;

R. Barbieri, M. Frigeni and F. Caravaglio, Phys. Lett. B 258 (1991) 395.

[23] R. Barbieri and G.F. Giudice, Nucl. Phys. B 306 (1988) 63.

[24] L. Roszkowski, Phys. Lett. B 278 (1992) 147.

[25] J. Lopez, D.V. Nanopoulos and K. Yuan, Phys. Lett. B 267 (1991) 219;

J. Lopez, D.V. Nanopoulos, and A. Zichichi, Phys. Lett. B 291 (1992) 255;

S. Kelley, J. Lopez, D.V. Nanopoulos, H. Pois and K. Yuan, CERN preprint CERN-TH-6584/92 (July 1992). 